\title{
TEMA 16-2015:Actualización en bronquiectasias
}

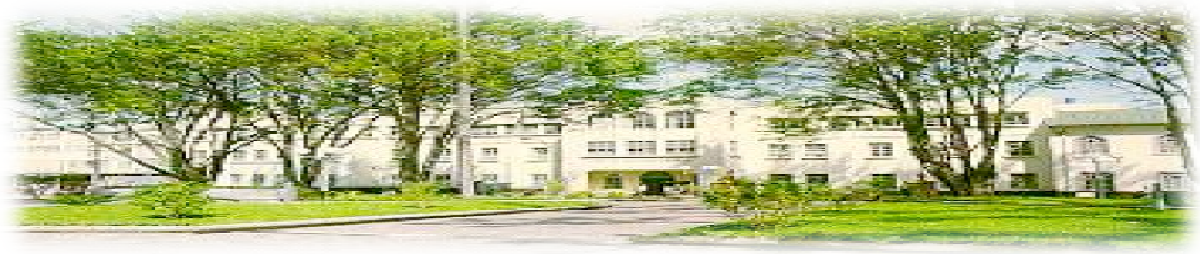

Hospital San Juan de Dios, San José, Costa Rica. Fundado en 1845
Recibido:

Aceptado:
$2 / 09 / 2015$

$25 / 09 / 2015$

Dra. Elizabeth Acón Ramírez ${ }^{1}$ Dr. Orlando Rodríguez Sánchez ${ }^{2}$

\footnotetext{
${ }^{1}$ Médico residente de Dermatología, CCSS-CENDEISSS, Hospital San Juan de Dios, eli.acon03@gmail.com

${ }^{2}$ Médico asistente especialista en Medicina Interna, Hospital San Juan de Dios, CCSS.
}

\section{RESUMEN}

Bronquiectasias se define como la dilatación bronquial irreversible que refleja la vía final común de una variedad de enfermedades subyacentes e insultos pulmonares no relacionados. Es una patología poco frecuente pero puede ser devastadora, con una alta tasa de morbimortalidad. Múltiples condiciones, congénitas o adquiridas predisponen al desarrollo de bronquiectasias, enfermedad que usualmente se presenta con tos productiva crónica y esputo viscoso. Datos demográficos, reportes de laboratorio, síntomas asociados y hallazgos en imágenes médicas guían el diagnóstico y dirigen hacia la sospecha de ciertas etiologías. El objetivo primordial del tratamiento consiste en limitar el círculo de infección-inflamación y por ende reducir el daño a la vía aérea, la sintomatología, el número de exacerbaciones y finalmente mejorar la calidad de vida.

\section{PALABRAS CLAVE}

Bronquiectasias, exacerbación, aclaramiento mucociliar, dilatación bronquial, remodelamiento de la vía aérea.

\section{ABSTRACT}

Bronchiectasis is the pathologic definition of irreversibly dilated bronchi and reflects the final common pathway of a variety of underlying diseases and unrelated pulmonary insults. It is a relatively uncommon but devastating disease associated with high morbidity and mortality. Multiple conditions, congenital or acquired predispose to bronchiectasis, disease that usually presents with chronic cough and daily viscid sputum production. Demographic data, laboratory results, associated symptoms and imaging findings guide the diagnosis and direct the suspicion to certain etiologies. The main goal of the treatment is to limit the cycle of infection and inflammation and, therefore, to reduce the air- 
way damage, chronic symptoms, number of exacerbations and finally to improve quality of life.

\section{KEY WORDS}

Bronchiectasis, exacerbation, airway drainage, bronchial dilation, airway remodeling.

\section{INTRODUCCIÓN}

Las bronquiectasias son dilataciones anormales y permanentes de los bronquios junto con alteración del epitelio ciliar. No corresponden a una enfermedad por sí mismas sino que representan el resultado final de distintas patologías con puntos de manejo comunes que suelen cursar con infección e inflamación bronquial crónica las cuales se ven asociadas con progresión. ${ }^{(1)} \mathrm{Si}$ bien son una entidad poco frecuente, pueden tener un curso devastador y verse asociadas a una alta morbimortalidad si no se diagnostican y tratan de manera temprana y adecuada. ${ }^{(2)}$

Se desconoce la prevalencia exacta en Estados Unidos y el resto del mundo, pero esta varía según el área geográfica. Son más comunes en el sexo femenino y la prevalencia aumenta conforme lo hace la edad. ${ }^{(3)}$

$\mathrm{Su}$ presentación clínica comparte características similares con la enfermedad pulmonar obstructiva crónica, incluyendo una vía aérea inflamada y fácilmente colapsable, obstrucción al flujo aéreo y frecuentes demandas de recursos en salud por exacerbaciones y hospitalizaciones recurrentes secundarias a sobreinfección; lo último en relación a la historia natural de la enfermedad que se caracteriza por fases de relativa estabilidad alternado con reagudizaciones y una tendencia general al deterioro de la función pulmonar con el tiempo. ${ }^{(4)}$ El diagnóstico se establece basado en la sintomatología típica de tos productiva con esputo mucopurulento diario asociado a imágenes médicas que evidencien dilatación del lumen bronquial y engrosamiento de la pared. ${ }^{(5)}$

\section{Fisiopatología}

El modelo fisiopatológico de la inducción de las bronquiectasias más ampliamente aceptado fundamenta su teoría en un círculo vicioso autoperpetuante en el cual una noxa infecciosa afecta a un huésped genéticamente susceptible o con una respuesta inmune deficiente, desencadenando un proceso inflamatorio complejo de la vía aérea con la consecuente retención mucosa y dificultad de aclaramiento mucociliar que predispone al individuo a colonización e infección crónica bronquial y termina causando la pérdida de la integridad estructural del mismo. ${ }^{(4)}$ Pese a que esta patología no se encuentra presente al nacimiento, existen múltiples factores congénitos y hereditarios que favorecen al desarrollo de bronquiectasias, incluyendo estados diversos de inmunodeficiencias, síndromes asociados a defectos genéticos tales como el síndrome de Mounier Kuhn, Williams Campbell, Kartagener, Young y la discinesia ciliar primaria. La propensión al desarrollo de bronquiectasias también puede ser adquirida representada por etiologías como obstrucción bronquial cualquiera que sea el origen, inhalación de tóxicos, enfermedades inflamatorias reumatológicas e intestinales, infección/aspiración recurrente, entre otras. Las causas pueden categorizarse como se ilustra en la tabla 1. ${ }^{(2)}$

El estímulo infeccioso es requisito primordial para el desarrollo de las bronquiectasias ya que corresponde al pivote desencadenante de la serie de eventos subsiguientes. Lo anterior debido a que al presentarse un patógeno, por lo general bacteriano en la vía aérea, se genera la activación de células efectoras inmunes, (predominantemente neutrófilos) que liberan proteasas, especies reactivas de oxígeno y otras citocinas inflamatorias con el fin de combatir la infección, sin embargo, a su vez lesionan el bronquio al producir inflamación transmural, edema asociado a ulceración mucosa, neovascularización $y$ en última instancia remodelamiento de la vía aérea con destrucción y dilatación anormal permanente de la pared del bronquio o bronquiolo. ${ }^{(6)}$ Dado este cambio en la arquitectura, los alveolos dependientes de la vía aérea afectada son hipoventilados, estímulo que genera anastomosis entre las circulaciones bronquial y pulmonar causando desequilibrio en la relación ventilación perfusión e hipoxemia, la cual si persiste puede llevar a hipertensión pulmonar y cor pulmonale. ${ }^{(7)}$

Cabe destacar también el papel potencial de la deficiencia de la vitamina D como factor contribuyente al desarrollo de bronquiectasias. Estudios comparativos entre pacientes con bronquiectasias y niveles deficientes de vitamina $\mathrm{D}$ versus 
pacientes que presentaban la misma patología pero niveles adecuados de la vitamina demostraron exacerbaciones más frecuentes, síntomas respiratorios más severos y una mayor tendencia a la colonización del esputo con gérmenes bacterianos potencialmente fatales incluyendo Pseudomonas aeruginosa en el primer grupo; sin embargo, las conclusiones no son claras respecto a si los hallazgos son explicados por la menor actividad al aire libre en los pacientes con enfermedad severa, o si verdaderamente la vitamina $D$ juega un papel significativo en la inmunidad innata. ${ }^{(8)}$

\section{Clasificación}

Existen distintos sistemas de clasificación de las bronquiectasias basados en patogénesis, etiología, factores predisponentes y hallazgos patológicos o anatómicos. El más reconocido y aceptado siendo la clasificación de Lynne Reid que las agrupa según la morfología y severidad. Este sistema anatómico las divide en tres grupos, en orden ascendente de severidad: ${ }^{(2,7)}$

1. Bronquiectasias tubulares o cilíndricas

Bronquios de contornos regulares, diámetro distal algo dilatado y la luz bronquialocluida por tapones mucosos.

2. Bronquiectasias varicosas

Reciben su nombre particular por la apariencia similar al de las venas varicosas.

Se caracterizan por la presencia de una vía aérea más dilataday de contornos irregulares por la presencia de constricciones fibrosas localizadas que le dan un aspecto irregular, tipo rosario.

3. Bronquiectasias saculares o quísticas

Presentan dilatación progresiva hacia la periferia de las vías aéreas y terminan en grandes quistes, sacos, o como racimos de uvas.

Cabe recalcar que si bien es cierto la clasificación anteriormente desglosada correlaciona de manera proporcional a la severidad clínica, su asociación con la etiología de las bronquiectasias es verdaderamente pobre y poco orientadora. ${ }^{(2,7)}$

\section{Clínica: Síntomas y hallazgos físicos}

Muchos de los pacientes pueden cursar asintomáticos e incluso no requerir tratamiento alguno; mientras que los que desarrollan sintomatología, por lo general tienen manifestaciones clínicas inespecíficas que varían según la etio-

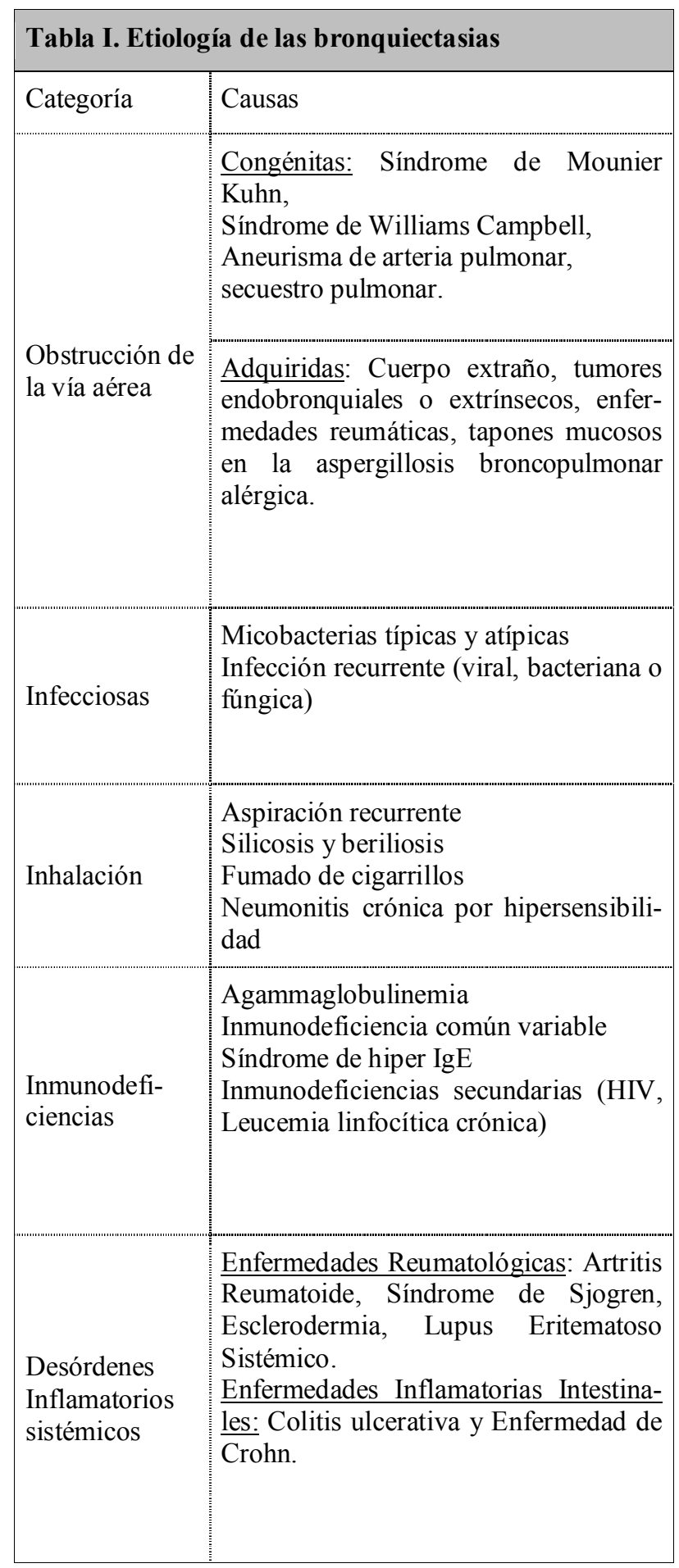

logía de fondo, comorbilidades individuales y severidad de la destrucción bronquial. ${ }^{(2)}$ 
La presentación típica es conocida como "bronquiectasias húmedas", lo cual incluye tos crónica con esputo mucoide, viscoso o mucopurulento que puede ser contínuo o recurrente posterior a un período de quiescencia. Se considera patognomónico, aunque no siempre presente, el esputo "de tres capas", caracterizado por una primera capa superficial espumosa, seguida de una mucoide medial y por último una capa viscosa purulenta. ${ }^{(2.7)}$

En el otro extremo, la presentación clínica puede darse como "bronquiectasias secas", en cuyo caso el paciente manifiesta tos no productiva y episodios de hemoptisis, explicados por la inflamación crónica bronquial que conlleva a hipertrofia y tortuosidad de las arterias bronquiales que acompañan el árbol bronquial regional al igual que la expansión del plexo vascular submucoso y peribronquial lo cual aumenta la susceptibilidad al sangrado. Pese a que esta no es la manifestación clínica usual, debe siempre considerarse dado que hasta el $50 \%$ de las bronquiectasias pueden complicarse con hemoptisis masiva de riesgo vital (definida como mayor a $100 \mathrm{cc}$ por hora o igual o mayor a $500 \mathrm{cc}$ por día); esto debido a que las arterias bronquiales se encuentran bajo presión arterial sistémica. De igual manera, sangrados escasos no deben despreciarse ya que son indicador confiable de infección y ameritan antibioticoterapia. Cabe mencionar que al presentarse un sangrado importante, siempre se debe descartar inicialmente que este provenga de vía respiratoria superior o que sea de origen gastrointestinal antes de asumir que es de origen pulmonar. ${ }^{(2)}$

Otros síntomas relacionados son disnea, dolor torácico pleurítico, fatiga generalizada, pérdida de peso y en la mitad de los pacientes puede desarrollarse sinusitis. ${ }^{(2,9)}$

Los hallazgos al examen físico igualmente son inespecíficos y consisten en: crépitos, sibilancias e hipocratismo digital en un 75, 22 y 2 por ciento respectivamente. ${ }^{(9)}$

\section{Abordaje Diagnóstico}

El propósito de la evaluación es confirmar el diagnóstico, identificar las potenciales causas tratables y la valoración funcional. Básicamente consiste en estudios de laboratorio, imágenes médicas y pruebas de función pulmonar. ${ }^{(2)}$

- Los exámenes de laboratorio deben enviarse orientados según la sospecha clínica etioló- gica. El hemograma completo es herramienta indispensable para identificar datos sugestivos de sobreinfección de las bronquiectasias y más si se asocia a cultivos de esputo. Los niveles de inmunoglobulinas IgA, IgM e IgG son también útiles ante la sospecha de inmunodeficiencias aunque el uso de las subclases de IgG como parámetro es controversial debido a que incluso en personas no enfermas el rango considerado normal fluctúa y es muy amplio, por lo que se recomienda medir niveles de anticuerpos basales y 46 semanas post vacunación (vacuna anti neumocócica) con el objetivo de valorar la respuesta inmune. Estudios genéticos como el análisis del gen del regulador de la conductancia transmembrana de la fibrosis quística pueden contribuir ante la sospecha de esta enfermedad. Anticuerpos específicos IgE e IgG contra Aspergillus, niveles totales de IgE , Alfa 1 antitripsina y factor reumatoide también pueden ser tomados en cuenta. (10-12)

- Respecto a las imágenes médicas, las bronquiectasias se presentan morfológicamente como bronquios dilatados, no afilados, de pared engrosada independientemente de los factores predisponentes y la etiología. La radiografía de tórax es un estudio bastante accesible y se documenta hallazgos patológicos en más del $80 \%$ de los pacientes con bronquiectasias, sin embargo no es un estudio sensible ni específico. Los signos radiográficos sugestivos comprenden el "rail en tranvía" que son opacidades lineales paralelas correspondientes a los bronquios dilatados de pared engrosada, el "anillo de se1lo", imagen del bronquio en que se observa bien definida la pared y por último el "dedo de guante" que representa opacidades irregulares periféricas correspondientes a tapones mucosos bronquiales. La presencia de dichos signos por separado y aún más en conjunto, soportan el diagnóstico si se ven asociados a clínica sugestiva. ${ }^{(2,14)}$

- El método de imagen estándar de oro para el diagnóstico es la tomografía axial computarizada de alta resolución en la cual los datos más específicos de bronquiectasias son la dilatación del bronquio, objetivizada como un diámetro bronquial mayor a 1.5 veces el diámetro de la arteria pulmonar adyacente, la falta de afilamiento bronquial que se ma- 
nifiesta como la presencia de estructuras bronquiales a menos de $10 \mathrm{~mm}$ de la pleura parietal y el patrón de árbol en gemación que indica afección de vía aérea pequeña. ${ }^{(14)}$ Además, la distribución de las bronquiectasias puede ser de gran relevancia para orientar el abordaje diagnóstico hacia una etiología en específico. Una distribución central es sugestiva de aspergillosis broncopulmonar alérgica, Síndrome de Mounier Kuhn o Síndrome de Williams Campbell. Lesiones en lóbulos superiores son frecuentes cuando la enfermedad de fondo es fibrosis quística o sus variantes, en lóbulo medio e inferior orientan hacia discinesia ciliar primaria, en lóbulos medio y língula es característico de micobacterias no tuberculosas y únicamente en lóbulos inferiores sugieren bronquiectasias idiopáticas. ${ }^{(15)}$

- La pruebas de función pulmonar, particularmente el volumen espiratorio forzado al primer minuto (VEF1) son un marcador confiable de la reserva pulmonar y la respuesta al tratamiento. Por lo general se documenta un patrón obstructivo con o sin evidencia de hiperreactividad de la vía aérea. ${ }^{(2,16)}$ Los volúmenes pulmonares se encuentran normales o disminuidos, la capacidad de difusión de monóxido de carbono con frecuencia está también disminuida pero con gradiente alveolo-arteriolar aumentado. ${ }^{(2)}$

\section{Tratamiento}

El objetivo del tratamiento del paciente con bronquiectasias es de una u otra manera bloquear el ciclo de infección - inflamación para de esta forma reducir el daño a la vía aérea, mejorar síntomas, disminuir reagudizaciones y así facilitar una mejor calidad de vida. ${ }^{(2)}$

Los pacientes con enfermedades congénitas que predispongan a bronquiectasias se consideran de alto riesgo y su manejo se basa en prevenir el daño adicional al bronquio mediante una adecuada nutrición, higiene de la vía aérea, promoviendo el buen aclaramiento de secreciones $\mathrm{y}$ aplicando las vacunas contra neumococo y virus influenza adicional al esquema tradicional de vacunación. ${ }^{(17,18)}$

Cabe destacar que el estudio de la etiología de fondo es necesario dado que el manejo adecuado de la causa subyacente permite limitar la progresión de la enfermedad. Típicamente es el caso de
Tabla II. Perfil microbiológico en reagudizaciones de bronquiectasias

Haemophilus influenzae tipo B

Pseudomonas aeruginosa

Streptococcus pneumoniae

Moraxella catarrhalis

Staphylococcus aureus

ciertas inmunodeficiencias primarias quienes responden al suministro de inmunoglobulina

purificada de plasma humano deteniendo el avance de las bronquiectasias; y también el manejo adecuado de la enfermedad por reflujo gastroesofágico puede tener el mismo efecto. ${ }^{(2,4,}$ 18,19)

El abordaje terapéutico de la enfermedad per sé debe subdividirse en el manejo de las crisis, definidas en base a la clínica (presencia/exacerbación de más de cuatro síntomas en un determinado momento) y no necesariamente en exámenes de laboratorio o imágenes; en segunda instancia debe considerarse la terapia de mantenimiento y prevención de exacerbaciones. ${ }^{(4)}$

En la mayoría de los casos los patógenos desencadenantes de las reagudizaciones son bacterianos, aunque el papel de los virus respiratorios no es despreciable. En la tabla 2 se exponen las bacterias más frecuentemente asociadas en en orden descendente en el caso de bronquiectasias no fibroquísticas. ${ }^{(4,20)}$

Por esta razón, las exacerbaciones se tratan con antibioticoterapia orientada según el germen aislado y la sensibilidad antibiótica del mismo. En el caso de no tener un esputo disponible, se debe iniciar tratamiento empírico con una fluoroquinolona. No existe un consenso en cuanto a la duración del mismo, sin embargo, en general se recomienda completar 10 días si es primera reagudización o pocas y de lo contrario, exacerbadores frecuentes o cuando el microorganismo aislado es una pseudomona, se deberá completar 14 días. ${ }^{(21,22)}$

Como medidas de mantenimiento tiene evidencia significativa el uso de antibiótico usualmente macrólido diario a bajas dosis por la vía oral y en 
presentación nebulizada fármacos antipseudomónicos. Agentes mucolíticos, soluciones hipertónicas y el manitol no se recomiendan de rutina y son necesarios mayores estudios para demostrar su beneficio. ${ }^{(2,4)}$

La cirugía se utiliza como último recurso en pacientes que no han mostrado respuesta satisfactoria al manejo médico y ha logrado disminuir significativamente la morbimortalidad en ciertos pacientes. ${ }^{(2)}$ El transplante pulmonar debe considerarse en pacientes jóvenes con bronquiectasias progresivas resistentes al manejo conservador y con una función pulmonar deficiente, sin embargo la sobrevida a 5 años es de sólo un $50 \%$. ${ }^{\text {(2) }}$

\section{BIBLIOGRAFÍA}

1. Vendrell M De Gracia J Olveira C et al. Diagnóstico y Tratamiento de las Bronquiectasias. Arch Bronconeumol. 2008; 44 (11): 629-40.

2. Oliva I Cortopassi F Herzog E et al. Clinical and Imaging Features of Bronchiectasis. Clin Pulm Med 2013; 20: 203-213.

3. Weycker D Eldesberg J Oster G Tino G. Prevalence and Economic burden of bronchiectasis. Clin Pulm Med 2005; 12:205.

4. Mc Donnell M Hester K De Soyza A. Bronchiectasis: Clinical Features and Management with a Focus on Inhaled Antibiotics. Clin Pulm Med 2014; 21: 251-261.

5. Barker AF. Bronchiectasis. N Engl J Med 2002; 346:1383.

6. Chalmers JD Hill AT. Mechanisms of immune dysfunction and bacterial persistence in noncystic fibrosis bronchiectasis. Mol Immunol 2013; 55:27.

7. Barberà JA Cosío MG. Bronquiectasias y otras enfermedades obstructivas de las vías aéreas. En: Farreras Valentí P, Rozman C, ed. Medicina interna. Edición 14a. Madrid: Hardcourt: 2000: vol 1: 860-869.

8. Chalmers JD McHugh BJ Docherty $\mathrm{C}$ et al. Vitamin $D$ deficiency is associated with chronic bacterial colonisation and disease severity in bronchiectasis. Thorax 2013; 68:39.

9. King PT Holdsworth SR Freezer NJ et al. Characterisation of the onset and presenting clinical features of adult bronchiectasis. Respir Med 2006; 100: 2183.

10. Bienvenu T Sermet-Gaudelus I Burgel PR et al. Cystic Fibrosis transmembrane conductance regulator channel dysfunction in non-cystic fibrosis bronchiectasis. Am J Respir Crit Care Med 2010; 181:1078.

11. Van de ven AA van Montfrans JM Terheggen-Lagro $\mathrm{SW}$ et al. A CT scan score for the assessment of lung disease in children with common variable immunodeficiency disorders. Chest 2010; 138:371.

12. Vendrell M De Gracia J Rodrigo MJ et al. Antibody production deficiency with normal $\operatorname{IgG}$ levels in bronchiectasis of unknown etiology. Chest 2005; 127:197.

13. Goeminne P Dupont L. Non-cystic fibrosis bronquiectasis: diagnosis and management in 21st century. Postgrad Med J. 2010; 86:493-501. 14. Gotaway M Gautham P Webb R et al. HighResolution CT of the Lung: Patterns of Disease and Differential Diagnoses.

15. Kim RD Greenberg DE Ehrmantraut ME et al. Pulmonary nontuberculous mycobacterial disease: prospective study of a distinct preexisting syndrome. Am J Respir Crit Care Med 2008; 178:1066.

16. Rowan SA Bradley JM Bradbury I et al. Lung clearance index is a repeatable and sensitive indicator of radiological changes in bronchiectasis. Am J Respir Crit Care Med 2014; 189:586.

17. Feldman C. Bronchiectasis: new approaches to diagnosis and management. Clin Chest Med. 2011; 32: 535-546.

18. Li AM Sonnappa $\mathrm{S}$ Lex $\mathrm{C}$ et al. Non-CF bronchiectasis: does knowing the aetiology lead to changes in management? Eur Respir J. 2005; 26:8-14.

19. Lucas M Lee M Lortan J et al. Infection outcomes in patients with common variable immunodeficiency disorders: relationship to immunoglobulin therapy over 22 years. J Allergy Clin Immunol 2010; 125:1354.

20. Gao Y Guan $\mathrm{W} X u \mathrm{G}$ et al. The role of viral infection in pulmonary exacerbations of bronchiectasis in adults: A prospective study. Chest 2014.

21. Pasteur MC Bilton D Hill AT. British Thoracic Society Bronchiectasis non-CF Guideline Group. British Thoracic Society Guideline for non-CF bronquiectasis. Thorax 2010;65 Supp11:11-

22. Murray MP Turnbull K Macquire S Hill AT. Assessing response to treatment of exacerbations of bronchiectasis in adults. Eur Respir J 2009; $33: 312$. 


\section{CONFLICTO DE INTERÉS YIO AGRADE-}

\section{CIMIENTOS}

Los autores declaran que no existió ningún conflicto de interés en el presente reporte. 